\title{
Developing a diagnostic method for latent tuberculosis infection using circulating miRNA
}

\author{
Shoji Hashimoto ${ }^{1}$, Hong Zhao ${ }^{2}$, Michiyo Hayakawa ${ }^{3}$, Koichi Nakajima², Y-h Taguchi ${ }^{4}$ and Yoshiki Murakami ${ }^{*}$
}

\begin{abstract}
Background: Mycobacterium tuberculosis is known to cause latent tuberculosis infection (LTBI) in $25-50 \%$ of the cases, of whom 10-20\% develop active tuberculosis (TB). Notably, no marker currently exists for judging the therapeutic effect of TB; it is currently judged by chest X-ray and clinical symptoms. We attempted to establish a marker for distinguishing LTBI from active TB and to identify the probability of recurrence after TB treatment, using information on circulating miRNA expression.

Methods: In total, 32 patients were enrolled in this study: 16 with an onset or recurrence of active TB, and 16 with LTBI showing positive interferon-gamma release assays (IGRA) test and chest $X$-ray. Total RNA from serum in an exosome-rich fraction was first extracted, followed by miRNA expression analysis using a next-generation sequencer, then, this data were analyzed using miRDeep2.

Results: Using the expression information of eight miRNAs, LTBI and TB could be diagnosed with an accuracy of 71.8\% (odds ratio: 6.16, $p$ value $=3.20 \mathrm{e}-02$ ) .

Conclusions: A novel method for efficiently differentiating between LTBI and active TB was established. This method appears to be promising for evaluating the therapeutic effect of $\mathrm{TB}$, as it can be performed in a minimally invasive manner.
\end{abstract}

Keywords: $L T B I, T B$, miRNA, Next-generation sequencing

\section{Background}

Tuberculosis (TB) is an infectious disease caused by Mycobacterium tuberculosis (Mtb) that affects the lungs. It is estimated that nearly $25 \%$ of the world's population is affected by TB [1]. The reason behind the uncontrollability of TB infection is not only poverty and coinfection with human immunodeficiency virus but also the lack of adequate information on the transmission and pathogenesis of this disease. Notably, nearly $5-15 \%$ of the people infected with TB develop overt infection, whereas most patients have latent tuberculosis infection (LTBI). However, patients with overt infection are at a risk of

\footnotetext{
* Correspondence: yoshikim@tokyo-med.ac.jp

${ }^{5}$ Department of Molecular Pathology, Tokyo Medical University, 6-1-1

Shinjuku, Shinjuku-ku, Tokyo 160-8402, Japan

Full list of author information is available at the end of the article
}

reactivation after completing their treatment [2]. Therefore, in TB treatment, it is important to diagnose LTBI and prevent the development of TB from LTBI. Once infected with $\mathrm{TB}$, most people remain asymptomatic and noncontagious. However, 5-15\% of cases with LTBI may progress to active TB during their lifetime and become infectious [3]. The risk of progression becomes even higher in younger children [4] and immunocompromised or immunosuppressed patients $[5,6]$.

Though for a long time tuberculin skin test (TST) had been used for the diagnosis of TB, now blood-based interferon-gamma release assays (IGRAs) are widely used to diagnose TB and LTBI because of their simplicity and high specificity [7], and positive IGRA test, normal chest X-ray and contact history (especially within 2 years in high prevalence area) are required to diagnose

(c) The Author(s). 2020 Open Access This article is licensed under a Creative Commons Attribution 4.0 International License, which permits use, sharing, adaptation, distribution and reproduction in any medium or format, as long as you give appropriate credit to the original author(s) and the source, provide a link to the Creative Commons licence, and indicate if changes were made. The images or other third party material in this article are included in the article's Creative Commons licence, unless indicated otherwise in a credit line to the material. If material is not included in the article's Creative Commons licence and your intended use is not permitted by statutory regulation or exceeds the permitted use, you will need to obtain permission directly from the copyright holder. To view a copy of this licence, visit http://creativecommons.org/licenses/by/4.0/. 
LTBI [8]. Moreover it is difficult to determine whether LTBI, a potential infectious disease has been successfully treated (prevented) $[9,10]$. Therefore, an accurate and convenient diagnostic method for LTBI has been desired.

Generally, miRNA is a noncoding type of RNA that has a length of about 20 bases, which regulates the expression of a target gene in a base-sequence-specific manner. Many researchers have reported that abnormal miRNA expression is related to the onset and exacerbation of many diseases for example, infectious diseases, degenerative diseases and cancer [11]. In addition, considering the fact that miRNAs are released extracellularly by cell-derived endoplasmic reticulum such as exosomes, miRNA in exosome reflects cellular information, and it is possible to obtain clinical information similar to that of collecting relevant organs [12]. Infection with Mtb first induces an interaction with macrophages, following which macrophages infected with Mtb release particular miRNAs packaged in exosomes into the extracellular space [13]. Several researchers have reported that many highly expressed miRNAs (miR-484, miR-425, and miR96) in exosomes are useful in the TB diagnosis [14]. However, this method for analyzing the miRNA profiles of TB and LTBI has not been applied in the diagnosis of LTBI [15].

Reducing the incidence of active $\mathrm{TB}$ requires new diagnostic tools and therapeutic options along with a better understanding of the microbiological and clinical spectrum of TB. In this study, we attempt to establish a diagnostic method using circulating miRNAs to develop a biomarker that can help easily evaluate the diagnostic efficiency and therapeutic effects of LTBI and active TB.

\section{Methods}

\section{Clinical background}

The study subjects comprised 16 patients with active TB, and 16 patients with LTBI (all above 18 years old) who visited or were hospitalized at the Osaka Habikino Medical Center for evaluation and initial treatment. This study was performed according to the principles of the Declaration of Helsinki. All study participants provided informed consent. Moreover, this clinical analysis was ethically reviewed and approved by the Osaka Habikino Medical Center (no. 773-4) and Osaka City University (no. 3747).

\section{RNA extraction and next-generation sequencing analysis}

Using $900 \mu \mathrm{L}$ of serum, an exosome-rich fraction was extracted using ExoQuick (System Biosciences, Palo Alto, CA, USA), and total RNA was extracted from a $200 \mu \mathrm{L}$ ExoQuick-treated solution using an RNeasy Mini Kit (Qiagen, Hilden, Germany). The concentration, integrity number, $28 \mathrm{~S} / 18 \mathrm{~S}$ ratio, and sample size of the extracted RNA were qualified using an Agilent 2100 Bioanalyzer (Agilent Technologies, Santa Clara, CA, USA).

To select an 18-30 nt stripe and recycle, total RNA was size-fractionated using polyacrylamide gel electrophoresis (PAGE) gel. An adaptor sequence was ligated with sizefractionated RNA and amplified. All PCR products were purified using PAGE, and then the recycled products were dissolved in an nuclease free water and he PCR product prepared was labeled to create a library. The library was then quantified, and the qualified libraries were sequenced using an Illumina HiSeq 4000 System (Illumina, San Diego, CA, USA). All sequencing data were deposited in NCBI's Gene Expression Omnibus and are accessible through the GEO accession number GSE149645.

\section{Statistics}

In this study, all miRNAs were selected using the recently proposed unsupervised feature extraction method based on tensor decomposition (TD) [16]. In total, 32 FASTQ files were mapped to the hg38 genome using miRDeep2 (https://www.mdc-berlin.de/content/mirdeep2-documentation) under default settings. The generated "miRNAs_ expressed_all_samples_X.csv" (where X is the time stamp) files were collected. Subsequently, the 32 expression profiles were formatted as a tensor, $x_{i j k} \in \mathbb{R}^{3017 \times 16 \times 2}$, which represents the expression of the $i$ th miRNA of the $j$ th normal controls $(k=1)$ or patients $(k=2)$. Notably, $x_{i j k}$ was normalized such that $\sum_{i} x_{i j k}=0$ and $\sum_{i} x_{i j k}^{2}=N$. Tensor decomposition was then obtained using higher-order singular value decomposition as follows:

$$
x_{i j k}=\sum_{l_{1} l_{2} l_{3}} G\left(l_{1}, l_{2}, l_{3}\right) u_{l_{1} j} u_{l_{2} k} u_{l_{3} i},
$$

where $G \in \mathbb{R}^{16 \times 2 \times 3017}$ is a core tensor and $u_{l_{1} j} \in \mathbb{R}^{16 \times 16}$, $u_{l_{2} k} \in \mathbb{R}^{2 \times 2}$, and $u_{l_{3} i} \in \mathbb{R}^{3017 \times 3017}$ are singular value orthogonal matrices. To identify the values of $u_{l_{3} i}$ used for miRNA selection, we identified which $u_{l_{3} i}$ represents patient- or normal-control-independent components and which $u_{l_{1} j}$ represents a distinction between healthy controls and normal controls. Subsequently, we verified whether $l_{1}=1$ and $l_{2}=2$ represent the required properties. We then tried to find the largest absolute value of $G\left(1,2, l_{3}\right)$, which we found to be $G(1,2,2)$. This means that $u_{2 i}$ should be used for miRNA selection. Therefore, the $p$-values were attributed to the $i$ th miRNA as follows:

$$
P_{i}=P_{X^{2}}\left[>\left(\frac{u_{2 i}}{\sigma_{2}}\right)^{2}\right],
$$

where $P_{\chi^{2}}[>x]$ is the cumulated $\chi^{2}$ distribution, in which the argument is larger than $x$, and $\sigma_{2}$ is the standard deviation. The $p$-values attributed to the $i$ th miRNA were 
corrected by the Benjamini-Hochberg criterion, and nine miRNA probes (hsa-let-7i-5p, miR-122-5p, miR-148a-3p, miR-151a-3p, miR-21-5p, miR-423-5p, miR-451a, and two miR-486-5p probes), associated with adjusted $p$ values less than 0.01 were selected. Then, the matrix $x_{i n} \in \mathbb{R}^{9 \times 32}$, which represents the $i$ th miRNA within the nine selected miRNAs of the $n$th patients/normal controls, was formatted. Then, $x_{i n}$ was normalized such that $\sum_{i} x_{i n}=0$ and $\sum_{i} x_{i n}^{2}=N$. Principal component analysis was then applied to $x_{i n}$ such that the principal component loading is attributed to the $n$th normal controls and patients. Then, the first and fourth principal component loadings were used to discriminate between the 16 normal controls and 16 patients. Linear discriminant analysis was also performed with two selected principal component loadings with leave-one-out crossvalidation, and all $p$-values were calculated using Fisher's exact test.

Table 1 Clinical background

\begin{tabular}{|c|c|c|c|c|c|c|c|}
\hline \multicolumn{5}{|c|}{ Basic patient information } & \multicolumn{3}{|c|}{ Diagnosis of TB } \\
\hline Sample ID & Diagnosis & Age & Sex & Complications & PCR & QFT & $\overline{\text { Sputum culture }}$ \\
\hline LP01 & LTBI & 44 & $\mathrm{~F}$ & & $(-)$ & 6.52 & $(-)$ \\
\hline LP02 & LTBI & 52 & M & & $(-)$ & 5.6 & $(-)$ \\
\hline LP03 & LTBI & 54 & $\mathrm{~F}$ & & $(-)$ & 2.03 & $(-)$ \\
\hline LP04 & LTBI & 52 & $\mathrm{~F}$ & & $(-)$ & 1.13 & $(-)$ \\
\hline LP05 & LTBI & 51 & $\mathrm{~F}$ & & $(-)$ & 0.87 & $(-)$ \\
\hline LP06 & LTBI & 61 & $\mathrm{~F}$ & & $(-)$ & 7.8 & $(-)$ \\
\hline LP07 & LTBI & 57 & $\mathrm{~F}$ & & $(-)$ & $>10$ & $(-)$ \\
\hline LP08 & LTBI & 24 & $\mathrm{~F}$ & & $(-)$ & 7.59 & $(-)$ \\
\hline LP09 & LTBI & 48 & M & & $(-)$ & $>10$ & $(-)$ \\
\hline LP10 & LTBI & 50 & $\mathrm{~F}$ & & $(-)$ & 2.45 & $(-)$ \\
\hline LP16 & LTBI & 78 & M & & NP & 0.34 & $(-)$ \\
\hline LP17 & LTBI & 57 & M & & NP & 2.38 & $(-)$ \\
\hline LP18 & LTBI & 67 & $\mathrm{~F}$ & & NP & 0.98 & $(-)$ \\
\hline LP22 & LTBI & 70 & M & Gastric cancer & NP & 2.45 & $(-)$ \\
\hline LP25 & LTBI & 75 & M & IP & NP & 9.5 & $(-)$ \\
\hline LP27 & LTBI & 81 & $\mathrm{~F}$ & & NP & 1.84 & $(-)$ \\
\hline TP01 & TB & 49 & $\mathrm{~F}$ & & $(-)$ & -0.05 & $(-)$ \\
\hline TP02 & TB & 56 & M & Colon cancer & NP & 2.23 & $(+)$ \\
\hline TP03 & TB & 62 & $\mathrm{~F}$ & Pleuritis & $(-)$ & 3.27 & $(-)$ \\
\hline TP04 & TB & 86 & M & & $(+)$ & NP & $(+)$ \\
\hline TP05 & TB & 83 & M & & $(+)$ & T-SPOT (+) & $(+)$ \\
\hline TP06 & TB & 75 & M & & $(-)$ & NP & $(-)$ \\
\hline TP07 & TB & 62 & M & $C L L$ & $(+)$ & T-SPOT (+) & $(+)$ \\
\hline TP08 & TB & 62 & M & Tongue cancer & $(+)$ & NP & $(+)$ \\
\hline TP09 & TB & 85 & $\mathrm{~F}$ & & NP & NP & $(+)$ \\
\hline TP10 & TB & 76 & M & Miliary TB & $(-)$ & NP & $(+)$ \\
\hline TP12 & TB & 35 & $\mathrm{~F}$ & & $(+)$ & NP & $(+)$ \\
\hline TP13 & TB & 29 & M & Lymph node TB & $\operatorname{LN}(+)$ & NP & $(-)$ \\
\hline TP17 & TB & 29 & $\mathrm{~F}$ & & $(+)$ & NP & $(+)$ \\
\hline TP25 & TB & 54 & $\mathrm{~F}$ & OC & $(+)$ & NP & $(+)$ \\
\hline TP33 & TB & 46 & $\mathrm{~F}$ & & $(+)$ & NP & $(+)$ \\
\hline TP41 & TB & 51 & M & DM & $(+)$ & NP & $(+)$ \\
\hline
\end{tabular}


Table 2 Diagnosis using miRNA information

\begin{tabular}{llll}
\hline & & Prediction & \\
& & LTBI & Active TB \\
Result & LTBI & 12 & 5 \\
& Active TB & 4 & 11 \\
\hline
\end{tabular}

\section{Results}

\section{Clinical background}

Among the study population, one patient with LTBI had gastric cancer and four patients with active TB had colon cancer, tongue cancer, chronic lymphocytic leukemia, and oral cancer. In addition, one patient with LTBI had interstitial pneumonia and one case of active TB had diabetes. On the other hand, in cases with active TB, extrapulmonary lesions were observed in two cases (miliary TB, lymph node TB; Table 1 and Supplementary Table 1).
Comparison of the expression of individual miRNAs

Using the expression information of eight miRNAs (hsalet-7i-5p, miR-122-5p, miR-148a-3p, miR-151a-3p, miR21-5p, miR-423-5p, miR-451a, and miR-486-5p), we were able to classify our patients into 16 patients with LTBI and 16 patients with active TB, with a diagnostic accuracy of $71.8 \%$ (odds ratio: 6.16, $p=3.20 \mathrm{e}-02$; Table 2).

Notably, four miRNAs (hsa-miR-122-5p, miR-151a-3p, miR-451a, and miR-486-5p) were found to exhibit a higher expression in LTBI than in active TB, with a significant difference observed in miR-122-5p $(p<0.05)$. On the other hand, four miRNAs were found to exhibit a higher expression in active TB than in LTBI (hsa-let7i-5p, miR-148a-3p, miR-21-5p, and miR-423-5p), which showed no significant difference in terms of expression (Fig. 1).

\section{Universally expressed novel miRNA candidates}

Novel miRNA candidates were analyzed using miRDeep2 (Fig. 2). In total, four types were expressed in all cases, with the expression of these four miRNA

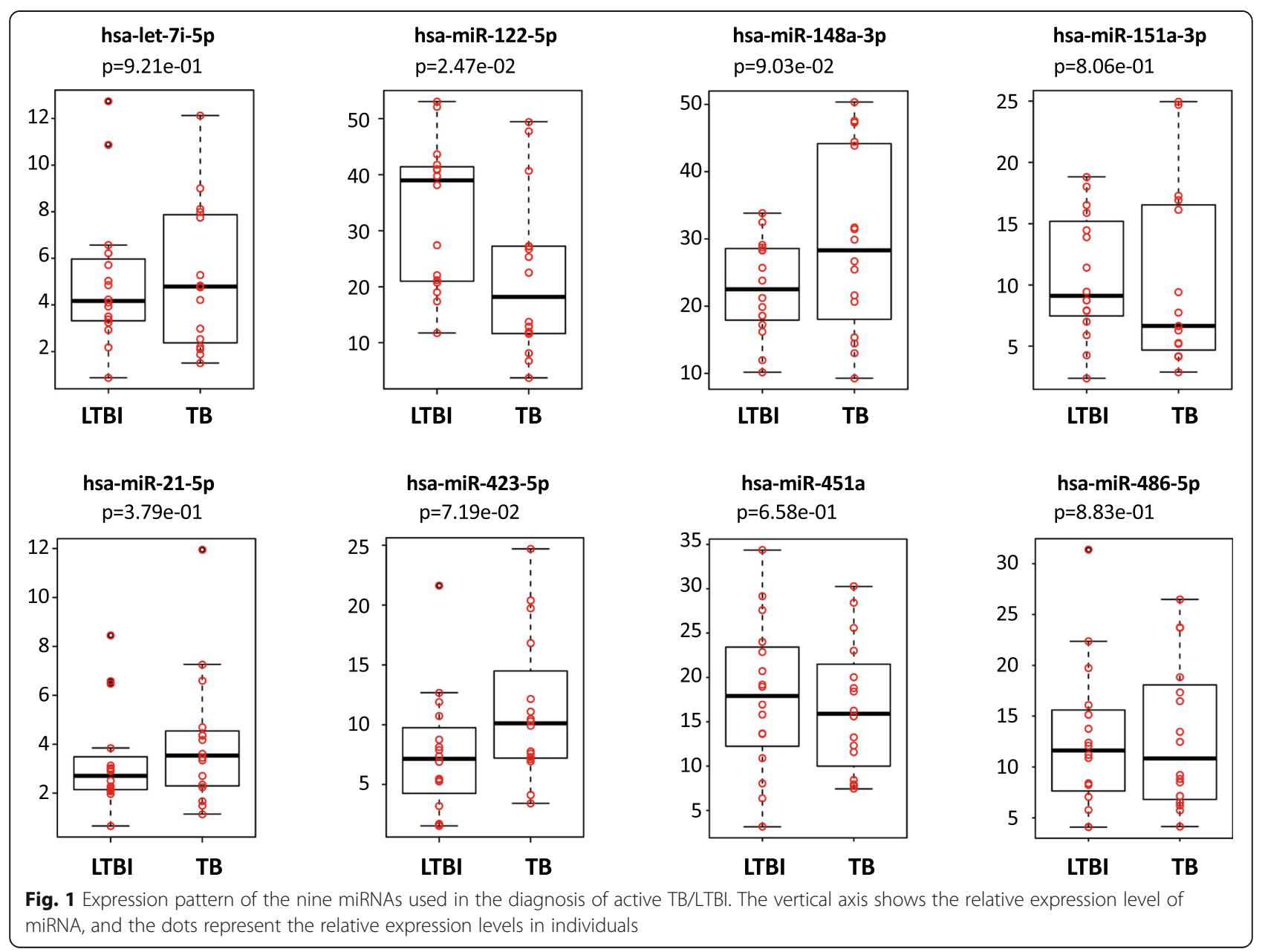




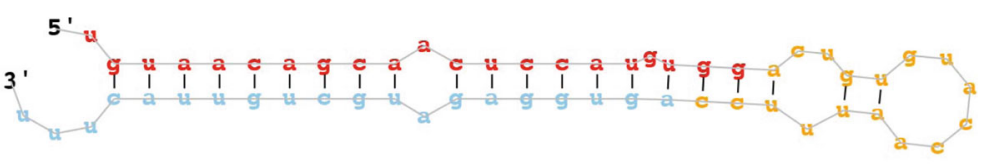

chr1_1669:chr1:220118169..220118227:-

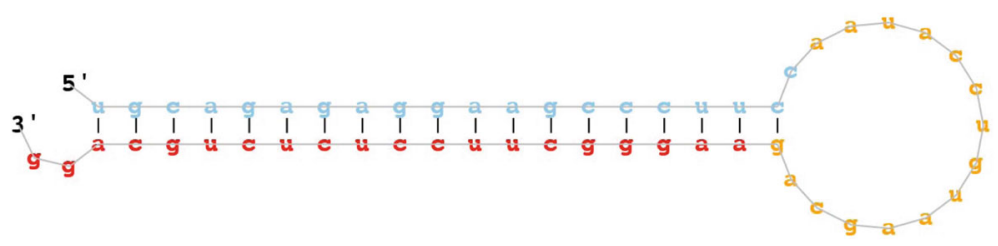

chr10_10988:chr10:101601428..101601483:-

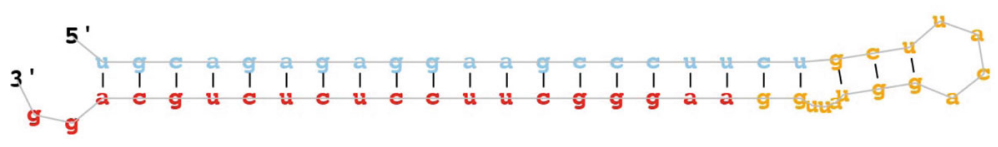

chr10_10553:chr10:101601430..101601485:+

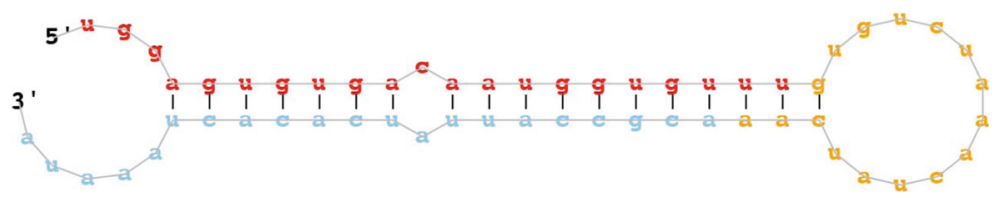

chr18_16140:chr18:58451087..58451145:+

Fig. 2 Virtual structure of novel miRNA candidates. The figure shows the structure of 11 miRNA candidates with significantly different expressions of $L T B I$ and active TB

candidates in LTBI being significantly higher than that in active TB $(p<0.05$; Table 3$)$.

\section{miRNA expression in airway-associated cells}

To examine the origin of miRNA in circulating blood, we compared the expression information of miRNA individually in the tracheal epithelial cells, bronchial epithelial cells, and alveolar epithelial cells of healthy subjects. The expression of miRNA was quoted from the miRNA Atlas of RIKEN (https://fantom.gsc.riken.jp/5/ suppl/De_Rie_et_al_2017/) [17]. As shown in Fig. 3, the expression of miR-122-5p and miR-451a is low, the expression of other miRNAs is abundant, and the expression of miR-21-5p in particular is enhanced. The
miRNAs of which expression level was high in TB as compared LTBI, were highly expressed in airway related cells. However, no significant differences were found in the expression of these miRNAs among the three types of epithelial cells mentioned above.

\section{Discussion}

Though IGRAs are considered to be useful for early diagnosis of LTBI and active TB instead of TST [7], differential diagnosis of LTBI and active TB is often difficult.

Patients with LTBI show positive IGRA test and normal chest X-ray. Oral isoniazid (INH) treatment for 6 months is considered an effective standard treatment to

Table 3 List of novel miRNA candidates showing ubiquitous expression

\begin{tabular}{|c|c|c|c|c|c|}
\hline \multirow{2}{*}{$\begin{array}{l}\text { Temporary miRNA name } \\
\text { (precursor coordinate) }\end{array}$} & \multicolumn{2}{|c|}{ Mature read count } & \multirow{2}{*}{$\begin{array}{l}\mathrm{LTBI} / \\
\mathrm{TP} \\
\text { ratio }\end{array}$} & \multirow[t]{2}{*}{$p$-value } & \multirow{2}{*}{$\begin{array}{l}\text { miRNA } \\
\text { with the } \\
\text { same seed }\end{array}$} \\
\hline & Average LTBI & Average TB & & & \\
\hline chr1:220118169..220118227:- & 1600.1 & 662.6 & 2.41 & $1.96 \mathrm{e}-03$ & ptr-miR-194 \\
\hline chr18:58451087..58451145:+ & $1,356,949.3$ & $482,662.4$ & 2.81 & $5.85 e-03$ & ptr-miR-122 \\
\hline chr10:101601428..101601483:- & 3652.0 & 1607.7 & 2.27 & $2.91 e-02$ & None \\
\hline chr10:101601430..101601485:+ & 3652.0 & 1607.7 & 2.27 & $2.91 e-02$ & None \\
\hline
\end{tabular}

Precursor coordinate: existing chromosome, gene start position and end position, sense strand (+), antisense strand (-). $p$-value: Student's $t$-test. miRNA with the same seed: a known miRNA in which the seed sequence of the new miRNA candidate is identical as analyzed by miRBase ver. 22.1 (www.mirbase.org) $L T B$ l latent tuberculosis infection, $T B$ tuberculosis 


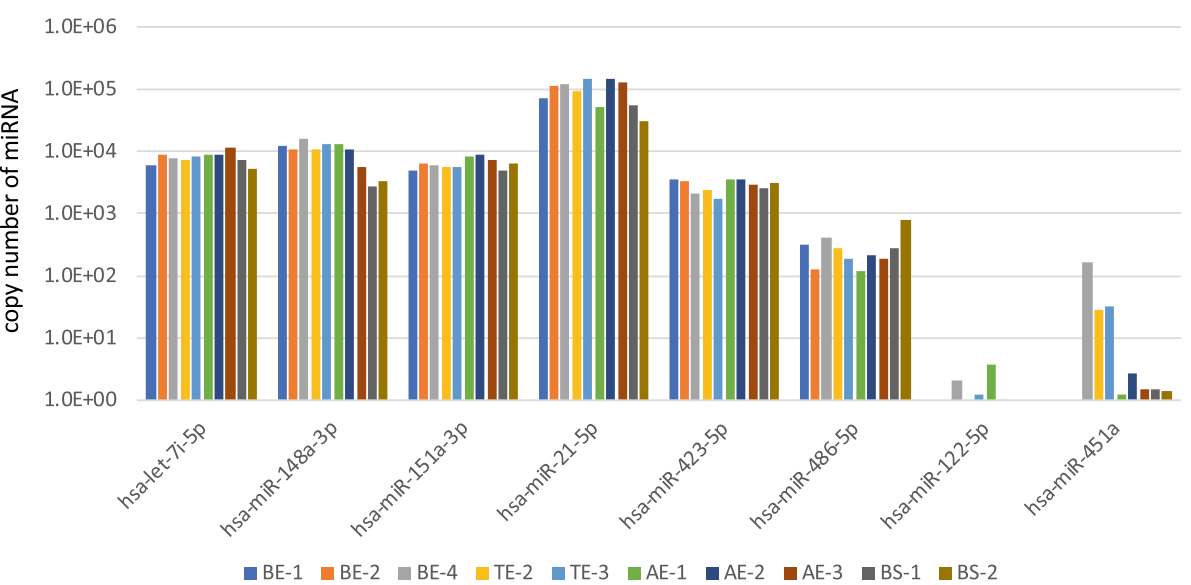

Fig. 3 miRNA expression levels in airway epithelial cells in next-generation sequencing. The figure shows the expression numbers of the eight miRNAs used in the diagnosis of active TB and LTBI in bronchial epithelium (BE), tracheal epithelium (TE), alveolar epithelium (AE), and bronchial smooth muscle (BS) cells. The miRNA expression profiles of the BE (BE-1, BE-2, and BE-4), TE (TE-2, TE-3), AE (AE-1, AE-2, and AE-3), and BS (BS-1, BS-2) cells were obtained from three, two, three, and two healthy volunteers, respectively

prevent the development of TB in LTBI patients infected within 2 years. Though oral anti-TB treatment reduces the incidence of active TB by about one-third [18], some patients with $\mathrm{TB}$, especially those who originally have liver dysfunction or heavy drinkers, develop liver dysfunction associated with TB treatment. In addition, LTBI and active TB due to INH-resistant Mtb increase the rate of treatment failure or relapse from 1.8\% [95\% confidence interval: $1.2-2.6$ ] to $10.9 \%$ [95\% confidence interval: 5.9-20] compared to Mtb susceptible to all anti-TB drugs [19]. Therefore, three evaluations are important: 1) whether the case is LTBI before the onset of TB, 2) there is no complicated liver dysfunction, and 3) the source of infection is not INH-resistant TB. Since the therapeutic effect cannot be judged using IGRA, it is judged by the improvement or deterioration of chest Xray and clinical symptoms.

Several reports exist on analysis using miRNA information from body fluids. When healthy individuals and individuals with active TB were diagnosed using miRNAs from exosomes derived from macrophages, an area under the receiver operating characteristic curve of 0.78 was obtained [13]. In addition, the information obtained from the six miRNAs (miR-20a, miR-20b, miR-26a, miR-106a, miR-191, miR-486) in plasma exosomes was useful for differentiating patients with tuberculous meningitis from those with TB [20]. Moreover, exosome miRNAs in pleural effusion were compared between three groups: an active TB group, a healthy control group, and a lung cancer group. Both miR-148a-3p and miR-451a were found to be highly expressed in the active TB group [21]. However, so far, no report exists on the differential diagnosis of active TB and LTBI.
Moreover, no particular correlation was found between circulating miRNA expression and miRNA expression in normal airway epithelial cells. It was also observed that the circulating expression of let-7i-5p, miR-148a-3p, miR-21-5p, and miR-423-5p, which are highly expressed in airway epithelial cells, is higher in cases with active TB than in cases with LTBI. However, the miR-122-5p and miR-451a were found to exhibit a higher expression in LTBI in circulating blood than in active TB. We also obtained multiple novel miRNA candidates that are expressed in all cases. Among these, the expression patterns of LTBI and active TB were found to be significantly different. Although the novel miRNA candidates need to be identified as miRNAs, they are considered as new biomarker candidates.

\section{Conclusions}

We attempted to differentiate LTBI from active TB using information on miRNAs in the circulating blood. Our results showed high diagnostic accuracy. Although the number of subjects in this study was limited, this novel method is promising not only for the differentiation between LTBI and active TB but also for evaluating the treatment effect for both, as compared to conventional IGRAs, chest X-ray, and clinical symptoms.

\section{Supplementary Information}

The online version contains supplementary material available at https://doi. org/10.1186/s41231-020-00078-7.

Additional file 1 : Supplementary Table 1. Clinical background in detail. 


\section{Abbreviations}

LTBI: Latent tuberculosis infection; TB: Tuberculosis interferon-gamma release assays (IGRA) test; Mtb: Mycobacterium tuberculosis; TST: Tuberculin skin test; PAGE: Polyacrylamide gel electrophoresis; TD: Tensor decomposition; INH: Isoniazid

\section{Acknowledgments}

The authors thank to Dr. Saori Itami-Matsumoto for preparing nucleic acid.

\section{Authors' contributions}

Hong Zhao, Michiyo Hayakawa, and Koichi Nakajima performed the experiments, contributed intellectual input, and provided the discussion. Shoji Hashimoto, Koichi Nakajima, and Y-h Taguchi helped write the manuscript and provided the discussion. Y-h Taguchi performed the nextgeneration sequencing analysis and data processing. Shoji Hashimoto and Yoshiki Murakami designed the experiments, analyzed and interpreted the data, and wrote the manuscript. The authors read and approved the final manuscript.

\section{Funding}

This research was supported by Grant-in-Aid for Scientific Research 16 K09037 (Hong Zhao, Koichi Nakajima, and Yoshiki Murakami) and 20K12067, 20H04848, and 19H05270 (Y-h. Taguchi).

\section{Availability of data and materials}

All data used in the manuscript will be provided upon request to the corresponding author.

\section{Ethics approval and consent to participate}

Not applicable.

\section{Consent for publication}

All the authors agree for the publication.

\section{Competing interests}

The authors have no competing interests to declare.

\section{Author details}

'Osaka Habikino Medical Center, Habikino, Osaka 583-8588, Japan. ${ }^{2}$ Department of Immunology, Osaka City University Graduate School of Medicine, Osaka 545-8585, Japan. ${ }^{3}$ Department of Pathology and Cell Regulation, Kyoto Prefectural University of Medicine, Kyoto 602-0841, Japan. ${ }^{4}$ Department of Physics, Chuo University, Tokyo 112-8551, Japan. ${ }^{5}$ Department of Molecular Pathology, Tokyo Medical University, 6-1-1 Shinjuku, Shinjuku-ku, Tokyo 160-8402, Japan.

Received: 23 November 2020 Accepted: 25 November 2020

Published online: 05 December 2020

\section{References}

1. Houben RM, Dodd PJ. The global burden of latent tuberculosis infection: a re-estimation using mathematical modelling. PLoS Med. 2016;13(10): e1002152.

2. Cadena AM, Fortune SM, Flynn JL. Heterogeneity in tuberculosis. Nat Rev Immunol. 2017:17(11):691-702

3. Diel $R$, Loddenkemper $R$, Nienhaus A. Predictive value of interferon-gamma release assays and tuberculin skin testing for progression from latent TB infection to disease state: a meta-analysis. Chest. 2012;142(1):63-75.

4. Kasprowicz VO, Churchyard G, Lawn SD, Squire SB, Lalvani A. Diagnosing latent tuberculosis in high-risk individuals: rising to the challenge in highburden areas. J Infect Dis. 2011;204(Suppl 4):S1168-78.

5. Santin M, Munoz L, Rigau D. Interferon-gamma release assays for the diagnosis of tuberculosis and tuberculosis infection in HIV-infected adults: a systematic review and meta-analysis. PLoS One. 2012;7(3):e32482.

6. Chkhartishvili N, Kempker RR, Dvali N, Abashidze L, Sharavdze L, Gabunia P, Blumberg HM, Del Rio C, Tsertsvadze T. Poor agreement between interferon-gamma release assays and the tuberculin skin test among HIVinfected individuals in the country of Georgia. BMC Infect Dis. 2013;13:513.

7. Auguste P, Tsertsvadze A, Pink J, Court R, McCarthy N, Sutcliffe P, Clarke A. Comparing interferon-gamma release assays with tuberculin skin test for identifying latent tuberculosis infection that progresses to active tuberculosis: systematic review and meta-analysis. BMC Infect Dis. 2017; 17(1):200.

8. Redelman-Sidi G, Sepkowitz KA. IFN-gamma release assays in the diagnosis of latent tuberculosis infection among immunocompromised adults. Am J Respir Crit Care Med. 2013;188(4):422-31.

9. Chee CB, KhinMar KW, Gan SH, Barkham TM, Koh CK, Shen L, Wang YT. Tuberculosis treatment effect on T-cell interferon-gamma responses to mycobacterium tuberculosis-specific antigens. Eur Respir J. 2010;36(2):35561.

10. Adetifa IM, Ota MO, Jeffries DJ, Lugos MD, Hammond AS, Battersby NJ, Owiafe PK, Donkor SD, Antonio M, Ibanga HB, et al. Interferon-gamma ELIS POT as a biomarker of treatment efficacy in latent tuberculosis infection: a clinical trial. Am J Respir Crit Care Med. 2013;187(4):439-45.

11. Rupaimoole R, Slack FJ. MicroRNA therapeutics: towards a new era for the management of cancer and other diseases. Nat Rev Drug Discov. 2017;16(3): 203-22.

12. Pos O, Biro O, Szemes T, Nagy B. Circulating cell-free nucleic acids: characteristics and applications. Eur J Hum Genet. 2018;26(7):937-45

13. Alipoor SD, Mortaz E, Tabarsi P, Farnia P, Mirsaeidi M, Garssen J, Movassaghi M, Adcock IM. Bovis Bacillus Calmette-Guerin (BCG) infection induces exosomal miRNA release by human macrophages. J Transl Med. 2017;15(1): 105.

14. Alipoor SD, Tabarsi P, Varahram M, Movassaghi M, Dizaji MK, Folkerts G, Garssen J, Adcock IM, Mortaz E. Serum Exosomal miRNAs are associated with active pulmonary tuberculosis. Dis Markers. 2019;2019:1907426.

15. Lyu L, Zhang X, Li C, Yang T, Wang J, Pan L, Jia H, Li Z, Sun Q, Yue L, et al. Small RNA profiles of serum exosomes derived from individuals with latent and active tuberculosis. Front Microbiol. 2019:10:1174.

16. Y-h T. Unsupervised feature extraction applied to bioinformatics: a PCA based and TD based approach. Switzerland: Springer international; 2020

17. de Rie D, Abugessaisa I, Alam T, Arner E, Arner P, Ashoor H, Astrom G, Babina M, Bertin N, Burroughs AM, et al. An integrated expression atlas of miRNAs and their promoters in human and mouse. Nat Biotechnol. 2017: 35(9):872-8

18. McMullan GS, Lewis JH. Tuberculosis of the liver, biliary tract, and pancreas. Microbiol Spectr. 2017;5(1). https://doi.org/10.1128/microbiolspec.TNMI70025-2016.

19. Stagg HR, Lipman MC, McHugh TD, Jenkins HE. Isoniazid-resistant tuberculosis: a cause for concern? Int J Tuberc Lung Dis. 2017;21(2):129-39.

20. Hu X, Liao S, Bai H, Wu L, Wang M, Wu Q, Zhou J, Jiao L, Chen X, Zhou Y, et al. Integrating exosomal microRNAs and electronic health data improved tuberculosis diagnosis. EBioMedicine. 2019:40:564-73.

21. Wang Y, Xu YM, Zou YQ, Lin J, Huang B, Liu J, Li J, Zhang J, Yang WM, Min $\mathrm{QH}$, et al. Identification of differential expressed PE exosomal miRNA in lung adenocarcinoma, tuberculosis, and other benign lesions. Medicine (Baltimore). 2017:96(44):e8361.

\section{Publisher's Note}

Springer Nature remains neutral with regard to jurisdictional claims in published maps and institutional affiliations.

Ready to submit your research? Choose BMC and benefit from:

- fast, convenient online submission

- thorough peer review by experienced researchers in your field

- rapid publication on acceptance

- support for research data, including large and complex data types

- gold Open Access which fosters wider collaboration and increased citations

- maximum visibility for your research: over $100 \mathrm{M}$ website views per year

At BMC, research is always in progress.

Learn more biomedcentral.com/submission 